19. Thompson CB, Eaton KA, Princiotta SM, Rushin CA, Valeri CR: Size-dependent platelet subpopulations: Relationship of platelet volume to ultrastructure, enzymatic activity, and function. Br J Haematol 50:509, 1982.

20. Dale J, Landmark KH, Nyhre E: The effects of nifedipine, a calcium antagonist, on platelet function. AM HEART J 105:103, 1983 .

21. Hiroki T, Inoue T, Yoshida T, Arakawa K: Effect of calcium antagonistic agent nifedipine on platelet aggregation response to exercise in patients with angina pectoris. Arzneim Forsch 32:1572, 1982.
22. Henry PD: Comparative pharmacology of calcium antagonists: Nifedipine, verapamil and diltiazem. Am J Cardiol 46:1047, 1980.

23. Pedersen OL, Mikkelsen E: Acute and chronic effects of nifedipine in arterial hypertension. Eur $\mathrm{J}$ Clin Pharmacol 14:375, 1978

24. Mehta P, Mehta J, Ostrowski N, Brigmon L: Inhibitory effects of diltiazem on platelet activation caused by ionophore A23187 plus ADP or epinephrine in subthreshold concentrations. J Lab Clin Med 102:332, 1983.

\title{
Postinfarction sudden death: Significance of inducible ventricular tachycardia and infarct size in a conscious canine model
}

The relationship between inducible ventricular tachycardia in the convalescent phase of myocardial infarction and subsequent spontaneous ventricular fibrillation is uncertain. Thirty conscious instrumented dogs underwent programmed ventricular stimulation 5 days after anterior infarction; 15 had inducible ventricular tachycardla and 15 were noninduclble. Following programmed ventricular stimulation, the application of a 150 uA current to the intima of the proximal circumflex artery initiated intimal damage, thrombosis, and acute ischemia of the posterolateral wall. After 20 minutes of ischemia, $73 \%$ inducible and $15 \%$ noninducible animals developed ventricular fibrillation $(p<0.005)$ without previous hypotension. At 24 hours, $7 \%$ inducible and $85 \%$ noninducible anlmals survlved $(p<0.001)$. Anterior infarct size (percentage of left ventricular mass) was much larger in inducible $(24.7 \pm 1.7 \%)$ than in noninducible $(5.3+1.1 \%)(p<0.001)$ animals. Inducible ventricular tachycardia following infarction was highly predictive of spontaneous ventricular fibrillation during a later ischemic episode in this model. The mass of previously injured myocardium was a critical determinant of both. (AM HEART J 109:8, 1985.)

David J. Wilber, M.D., Joseph J. Lynch, Ph.D., Daniel Montgomery, and Benedict R. Lucchesi, Ph.D., M.D. Ann Arbor, Mich.

Over one half of all sudden deaths occur in patients with prior myocardial infarction, ${ }^{1-3}$ with the majority occurring in the first few months after infarction..$^{4,5}$ Left ventricular dysfunction and frequent ventricu-

From the Departments of Pharmacology and Internal Medicine, University of Michigan Medical School.

Supported by grants from the National Institutes of Health, Heart, Lung, and Blood Institute, HL-19782-06 and HL-05806-23, and by a Postdoctoral Fellowship of the Michigan Heart Association (Dr. Lynch).

Received for publication June 22, 1984; accepted July 27, 1984.

Reprint requests: Benedict R. Lucchesi, Ph.D.,M.D., Department of Pharmacology, M6322 Medical Science Building I, The University of Michigan Medical School, Ann Arbor, MI 48109. lar ectopy are present in the majority of those who will subsequently die suddenly. ${ }^{6-8}$ However, the presence of these chronic abnormalities fails to explain why particular individuals experience fatal arrhythmias at specific points in time. Recent evidence suggests that patients who demonstrate recurrent, transient episodes of ischemia after infarction, either at rest or with exercise, are at higher risk of later sudden death. Schuster and Bulkley ${ }^{9}$ found a $27 \%$ incidence of sudden death over an average follow-up of 3 months in patients who manifested postinfarction angina during their early convalescence. Theroux et al. ${ }^{10}$ studied 210 patients without 
angina or clinical heart failure by submaximal exercise testing 2 weeks after infarction. Patients with 1 $\mathrm{mm}$ or more of ST segment depression during exercise had a $15 \%$ incidence of sudden death at 1 year compared to less than $1 \%$ incidence among those without ST depression. Acute ischemia therefore may be an important trigger event for sudden death in many of these patients.

In an attempt to refine the identification of high-risk patients and to direct preventive therapy, several groups of investigators have applied the techniques of programmed ventricular stimulation to patients in the early convalescent phase of infarction. Initial studies indicated a relatively high predictive value of inducible ventricular tachycardia for sudden death over the following year. ${ }^{11}{ }^{12}$ Subsequent reports have been less encouraging. However, significant differences exist in patient selection, induction protocol, criteria for a positive response, and timing of study after the index infarction, precluding definitive conclusions. ${ }^{13-15}$ Recently, this laboratory has developed a conscious canine model for the study of sudden death precipitated by an episode of acute ischemia superimposed upon chronic infarction. ${ }^{16}$ In this investigation we examine the characteristics of the convalescent postinfarct dog, including the response to programmed stimulation, that influence the likelihood of ventricular fibrillation following a subsequent ischemic episode.

\section{METHODS}

Surgical preparation. Male mongrel dogs, weighing 14 to $20 \mathrm{~kg}$, were anesthetized with sodium pentobarbital, 30 $\mathrm{mg} / \mathrm{kg}$, and ventilated on room air with a Harvard respirator via a cuffed endotracheal tube. By means of aseptic techniques, a left thoracotomy was performed in the fourth intercostal space. The pericardium was opened and the heart was suspended in a pericardial cradle. The left anterior descending coronary artery was isolated at the tip of the left atrial appendage. A stenosis was produced by placing a ligature around both the artery and a 20 -gauge hypodermic needle, and subsequently withdrawing the needle. Previous work has suggested that this degree of stenosis is sufficient to limit reactive hyperemia in response to a brief ( 10 second) coronary occlusion. ${ }^{16}$ The objectives in producing the stenosis were to limit arrhythmias immediately upon reperfusion ${ }^{17}$ and to more closely mimic the clinical situation where therapeutic or spontaneous reperfusion occurs in the setting of significant residual coronary stenosis. The artery then was occluded for 2 hours by means of a share formed with a loop of silicone rubber tubing passed through a polyethylene tube. Return of blood flow was assured in all instances by visible distention of the distal artery upon release of the snare and the return of normal color to the epicardium of the distal perfusion bed.

The left external jugular vein and the left common

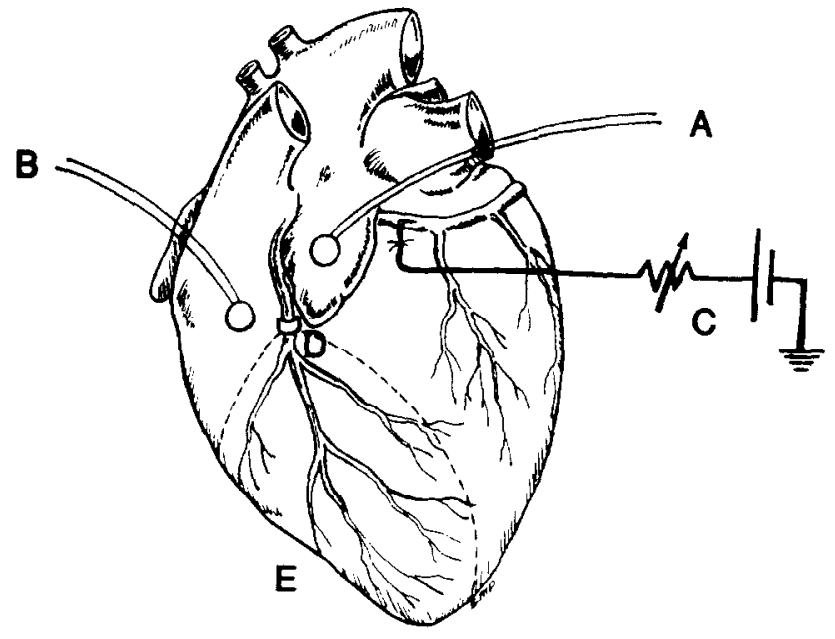

Fig. 1. Representation of the surgical preparation, demonstrating the placement of atrial $(A)$ and ventricular $(B)$ bipolar stimulating electrodes. The circumflex intimal electrode $(C)$ is attached at a later time to the positive pole of a 9-volt nickel-cadmium battery and variable resistor; the negative pole is a subcutaneous electrode. A critical left anterior descending artery stenosis $(D)$ remains in place after production of anterior infarction $(E)$ by 2-hour occlusion at the site of stenosis.

Table I. Baseline characteristics of the conscious canine model 4 to 6 days after myocardial infarction

\begin{tabular}{lcc}
\hline \multicolumn{1}{c}{ Characteristics } & $\begin{array}{c}\text { Inducible } \\
(n=15)\end{array}$ & $\begin{array}{c}\text { Noninducible? } \\
(n=15)\end{array}$ \\
\hline Weight (kg) & $17.4 \pm 0.2$ & $16.0 \pm 0.4$ \\
Heart rate (bpm) & $114 \pm 6$ & $113 \pm 6$ \\
Mean arterial pressure & $93 \pm 3$ & $90 \pm 5$ \\
QRS duration (msec) & $56 \pm 1$ & $54 \pm 1$ \\
PR interval (msec) & $108 \pm 5$ & $116 \pm 5$ \\
QT interval (msec) & $302 \pm 10$ & $300 \pm 11$ \\
Excitability threshold (volts) & $2.4 \pm 0.2$ & $2.5 \pm 0.3$ \\
Effective refractory period & $140 \pm 4$ & $145 \pm 6$ \\
$\quad$ (msec) & & \\
Anterior infarct size (c) of & $24.7 \pm 1.7^{*}$ & $5.3 \pm 1.1^{*}$ \\
$\quad$ left ventricular mass) & &
\end{tabular}

${ }^{*}$ Difference significant, $p<0.001$.

carotid artery were isolated and cannulae inserted; these were exteriorized to the back of the neck through a subcutaneous tunnel. A silver bipolar electrode $(1 \mathrm{~mm}$ diameter, $3 \mathrm{~mm}$ separation) was sutured to the left atrial appendage. A bipolar stainless steel plunge electrode (25 gauge, $5 \mathrm{~mm}$ length, $2 \mathrm{~mm}$ separation) was sutured to the right ventricular outflow tract adjacent to the intraventricular septum. The distal $3 \mathrm{~mm}$ of insulated 30 -gauge silver wire was bared and inserted into the lumen of the left circumflex coronary artery parallel to the vessel. The wire was sutured in place on the adjacent epicardial surface. The pericardium was closed loosely. Subcutaneous silver disc electrodes were implanted and all wires were exteriorized. The incision was closed and the animal was 

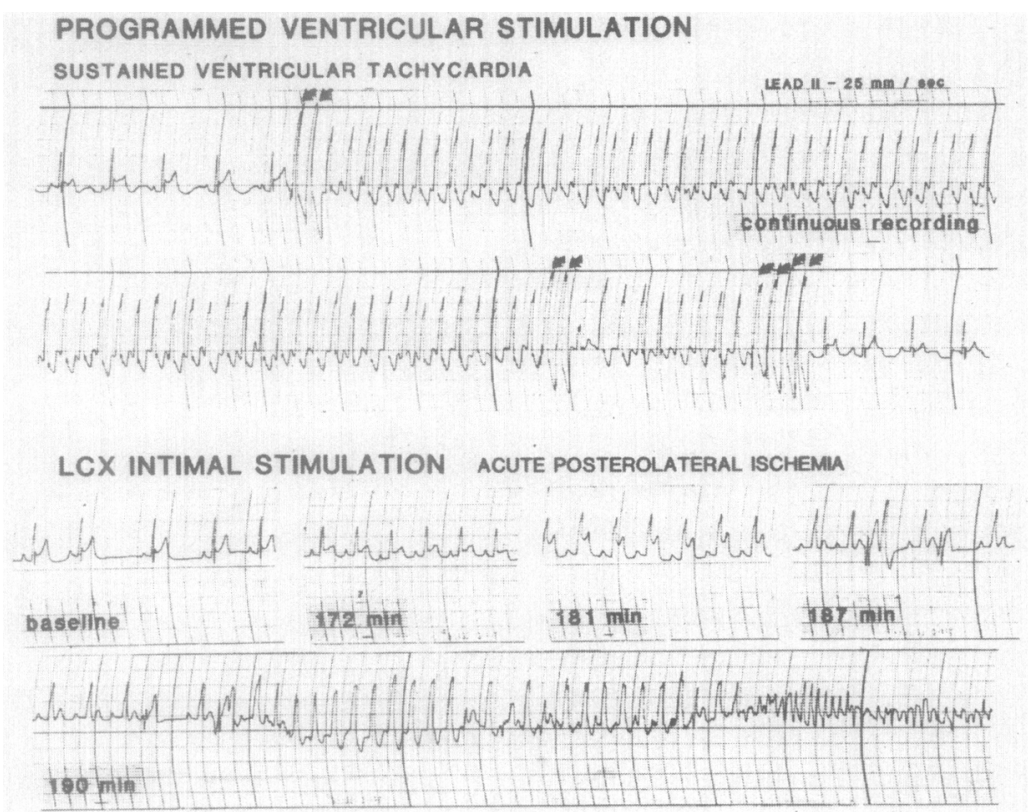

Fig. 2. Upper, Programmed stimulation in a dog with a large anterior infarction (30\% left ventricle). Sustained ventricular tachycardia (rate 255) was induced by double extrastimuli at coupling intervals of 250 and $180 \mathrm{msec}$, respectively (arrows) and could be terminated by four, but not two, extrastimuli. Lower, Onset of ST elevation occurred after 172 minutes of current flow through the circumflex electrode. After 15 minutes of ischemia, frequent premature ventricular contractions appeared followed by ventricular tachycardia at a similar rate and morphology to the induced arrhythmia. The tachycardia rapidly accelerated and degenerated into fibrillation.

allowed to recover from anesthesia. Fig. 1 illustrates various features of the experimental preparation.

Programmed ventricular stimulation in the convalescent phase. Programmed ventricular stimulation was performed 4 to 6 days after anterior myocardial infarction. All animals were in sinus rhythm without evidence of spontaneous ectopy. The animals were studied in the conscious state without sedation and while resting comfortably in a sling. The lead II ECG and aortic pressure were recorded continuously on a Grass polygraph. Atrial pacing was performed by means of a Grass model S-44 stimulator and a Grass model PSIU-6 stimulus isolation unit. Premature ventricular stimuli were introduced by means of a Grass model S- 88 stimulator and a Grass model SIU-5 stimulus isolation unit.

After recording of baseline ECG and hemodynamic measurements, excitability thresholds were determined by the introduction of single premature stimuli at a coupling interval of $300 \mathrm{msec}$ during atrial pacing at a cycle length of $400 \mathrm{msec}$, using incremental voltages until capture occurred. Ventricular refractoriness was determined by the introduction of single extrastimuli (twice threshold, 4 msec duration) during atrial pacing at the same cycle length. The coupling interval of the extrastimulus $\left(\mathbf{S}_{2}\right)$ to the basic rhythm $\left(\mathbf{S}_{1}\right)$ was decreased progressively from $300 \mathrm{msec}$ until capture failed to occur. Double extrastimuli $\left(\mathrm{S}_{2}-\mathrm{S}_{3}\right)$ then were introduced during sinus rhythm. The initial $S_{1}-S_{2}$ interval was set at $50 \mathrm{msec}$ greater than the refractory period, and the initial $S_{2}-S_{3}$ interval was set at twice the $S_{1}-S_{2}$ interval. The $S_{2}-S_{3}$ interval was shortened progressively by $10 \mathrm{msec}$ intervals until $S_{3}$ failed to capture. The $S_{2}$ then was moved closer to $S_{1}$ and the $S_{2}-S_{3}$ was again shortened by $10 \mathrm{msec}$ decrements. The entire sequence was repeated until $\mathrm{S}_{2}$ failed to capture. Triple extrastimuli $\left(\mathrm{S}_{2}-\mathrm{S}_{3}-\mathrm{S}_{4}\right)$ were then introduced in a similar fashion. The entire protocol was repeated during atrial pacing at cycling lengths of 350 to $400 \mathrm{msec}$. Finally, burst ventricular pacing was performed at cycle lengths of 300 to $600 \mathrm{msec}$.

The end points of programmed stimulation were the reproducible induction of ventricular tachycardia or completion of the protocol. Nonsustained ventricular tachycardia was defined as five or more repetitive beats, which terminated spontaneously within 30 seconds. Ventricular tachycardia was considered sustained if it persisted at least 30 seconds or, in the event of hemodynamic compromise, required pacing techniques for termination. If nonsustained ventricular tachycardia could be initiated repeatedly, more aggressive attempts to induce sustained tachycardia were not made. Animals responding with less than five nonstimulated depolarizations during the entire protocol were designated as being noninducible. If ventricular tachycardia degenerated into ventricular fibrillation before being terminated by pacing, the animal was excluded from the study to avoid the potentially confounding influence of occasionally prolonged resuscitative 


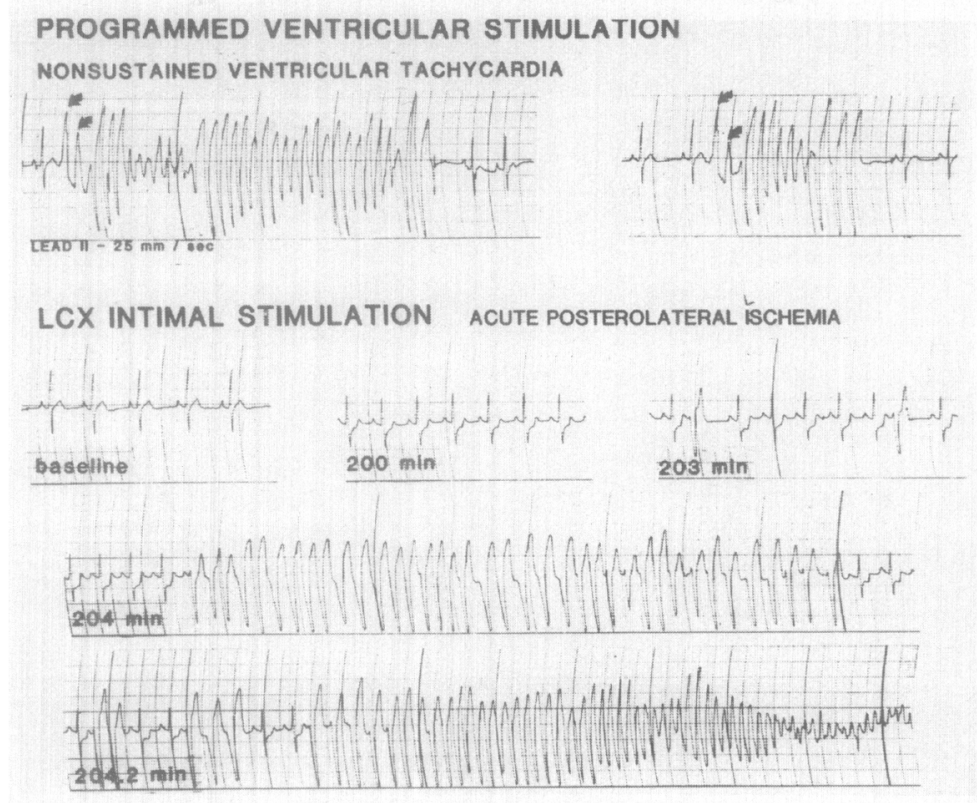

Fig. 3. Upper, Nonsustained ventricular tachycardia was induced by double extrastimuli (coupling intervals of 230 and $150 \mathrm{msec}$ ) in an animal with a large anterior infarct (19.5\% left ventricle). Lower, The onset of ST depression and sinus tachycardia occurs after 200 minutes of current flow through the circumflex electrode, followed by frequent premature ventricular contractions and a self-terminating run of ventricular tachycardia. A final episode of ventricular tachycardia preceded fibrillation after 4 minutes of ischemia.

efforts on the outcome of the second phase of the investigation.

Acute posterolateral ischemia in the convalescent phase. After completion of programmed ventricular stimulation, an anodal direct current of $150 \mathrm{uA}$ was applied to the intima of the circumflex coronary artery via the previously implanted silver wire. The lead II ECG was recorded continuously until death or for 24 hours after the onset of ECG evidence of acute ischemia, either directly on a Grass polygraph or on a cardiocassette FM recorder.

At the time of death, or after 24 hours of ischemia, the heart was excised. The proximal circumflex coronary artery was examined for proper placement of the intraluminal wire electrode and evidence of intimal injury; intravascular thrombus was removed and weighed. The heart was sectioned transversely into 5 to $10 \mathrm{~mm}$ slices and then incubated in $0.5 \%$ triphenyltetrazolium chloride and $0.01 \%$ phosphate buffer ( $\mathrm{pH} 7.4$ ). Normal myocardium stains an intense red and is demarcated clearly from irreversibly injured myocardium. ${ }^{18}$ Infarct mass and total left ventricular mass were quantitated gravimetrically. Infarct size was expressed as a percentage of the total left ventricular mass.

Experimental design and statistical analysis. Animals not excluded because of ventricular fibrillation during programmed stimulation were entered into the study until 15 inducible and 15 noninducible animals could be compared. Descriptive data are expressed as mean \pm standard error. Differences between group means were analyzed by the unpaired Student's $t$ test. Differences in survival at particular points in time were analyzed by a Fisher's exact test. The relationship between posterolateral and anterior infarct size in surviving animals was examined by linear regression analysis.

\section{RESULTS}

Programmed stimulation in the convalescent phase. On the day of study, inducible and noninducible animals were similar with respect to baseline hemodynamic, ECG, and electrophysiologic parameters (Table I). Of the 15 inducible animals, eight had sustained ventricular tachycardia (rate $364 \pm 26$ ventricular complexes/minute), reproducibly initiated by single $(n=1)$, double $(n=5)$, or triple $(\mathbf{n}=2)$ extrastimuli. In all cases, ventricular tachycardia was terminated by one to five extrastimuli (Fig. 2, Upper panel). Nonsustained ventricular tachycardia (11 \pm 3 nonstimulated beats) was initiated in seven animals by single $(n=1)$, double $(n=3)$, or triple $(n=3)$ extrastimuli (Figs. 3 and 4$)$. Animals with sustained and those with nonsustained ventricular tachycardia had identical baseline characteristics. None of the 15 noninducible animals responded with more than three nonstimulated complexes during the entire protocol.

Acute posterolateral ischemia in the convalescent phase. After the completion of programmed stimula- 


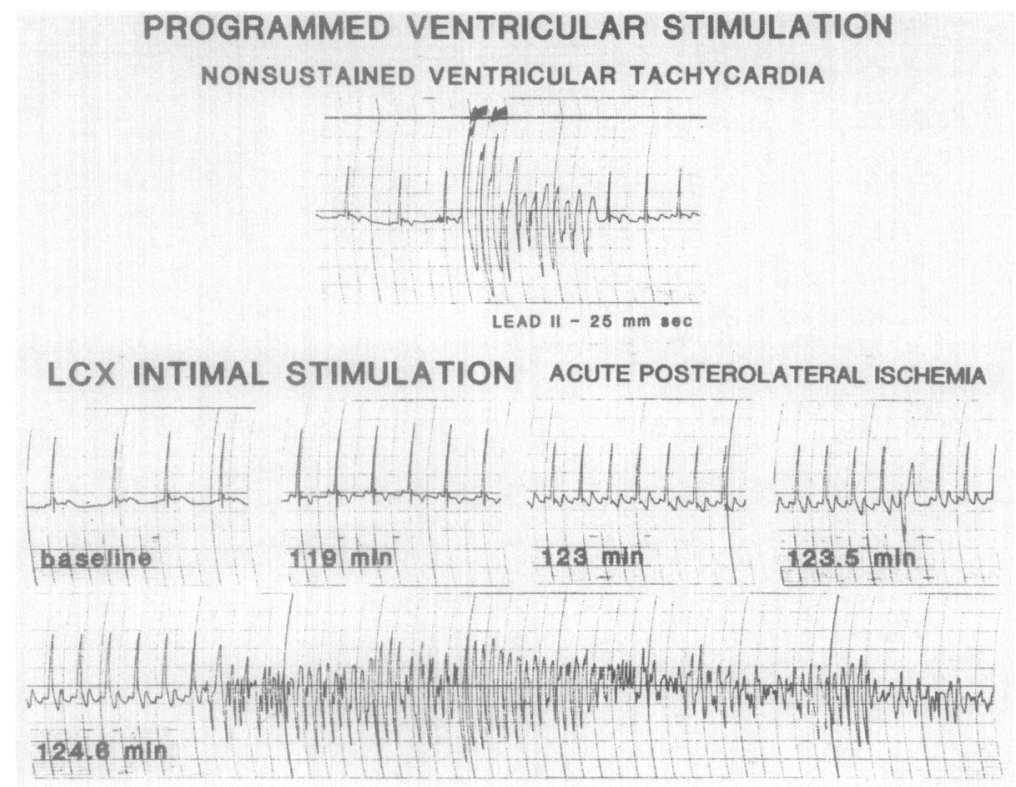

Fig. 4. Upper, Nonsustained ventricular tachycardia was induced by double extrastimuli (coupling intervals of 180 and $160 \mathrm{msec}$ ) in an animal with a large anterior infarction (29\% left ventricle). Lower, During 5 minutes of ischemia prior to death, only a single premature ventricular contraction preceded the onset of ventricular flutter.

Table II. Acute posterolateral ischemia in the convalescent phase of anterior myocardial infarction

\begin{tabular}{lcc}
\hline & $\begin{array}{c}\text { Inducible } \\
(n=15)\end{array}$ & $\begin{array}{c}\text { Noninducible } \\
(n=15)\end{array}$ \\
\hline $\begin{array}{c}\text { Time to ST segment change } \\
\text { (min) }\end{array}$ & $196 \pm 39$ & $225 \pm 30$ \\
$\begin{array}{c}\text { Peak heart rate after ST } \\
\text { segment change }\end{array}$ & $159 \pm 6$ & $166 \pm 12$ \\
$\begin{array}{c}\text { Early ventricular } \\
\text { fibrillation* }\end{array}$ & $11 / 15$ & $2 / 15$ \\
$\begin{array}{c}\text { Posterolateral infarct size } \\
\text { (\% left ventricular } \\
\text { mass) }\end{array}$ & $\begin{array}{c}19 \pm 1 \\
(\mathrm{n}=3)\end{array}$ & $\begin{array}{c}16.7 \pm 3.1 \\
(\mathrm{n}=13)\end{array}$ \\
Thombus mass (mg) & $7.2 \pm 1.8$ & $11.2 \pm 2.3$ \\
\hline
\end{tabular}

*Within 20 minutes of onset of ST segment changes; difference significant, $p<0.005$.

tion, the application of an anodal current to the intraluminal circumflex coronary artery electrode was followed by ECG evidence of posterolateral ischemia in leads II, III, and/or $\mathrm{aV}_{\mathrm{F}}$ at similar time intervals for inducible and noninducible animals (Table II). The maximum heart rate attained after the onset of ischemia also was similar in both groups. Within 20 minutes of the onset of ischemia, $73 \%$ of inducible animals and only $13 \%$ of noninducible animals had developed ventricular fibrillation $(p<0.005)$. None of the animals had significant hypotension (systolic pressure less than $90 \mathrm{~mm}$ ) or heart block preceding their fatal arrhythmia.
The ECG events culminating in ventricular fibrillation were variable. Both survivors and nonsurvivors manifested ventricular ectopy, ranging from one or two ventricular complexes to frequent multiform premature ventricular complexes and runs of nonsustained ventricular tachycardia (Figs. 2 to 5). Because of this wide variability, no attempt was made to quantitate the frequency of ectopic beats. In all experiments, ventricular fibrillation was preceded by a more organized ventricular rhythm, which lasted for a few cycles to several seconds before terminating in ventricular fibrillation. Two patterns emerged. In the first, the initial ventricular complexes were uniform and had relatively long coupling intervals. After a variable interval, the rhythm accelerated and became progressively more chaotic. The initial run of ventricular tachycardia occasionally (Fig. 2), but not invariably (Fig. 3), resembled the previously induced tachycardia in rate and morphology. In other animals the initial rhythm was a rapid polymorphic ventricular tachycardia or flutter (Fig. 4).

No late deaths occurred in the noninducible group, while three late episodes of ventricular fibrillation (at 3,13, and 16 hours after ischemia) occurred in the inducible group. Animals that survived generally developed striking ventricular ectopy after several hours of quiescence; after 24 hours, frequently $>50 \%$ of all complexes were ventricular in origin. Rapid or polymorphic ventricular tachy- 


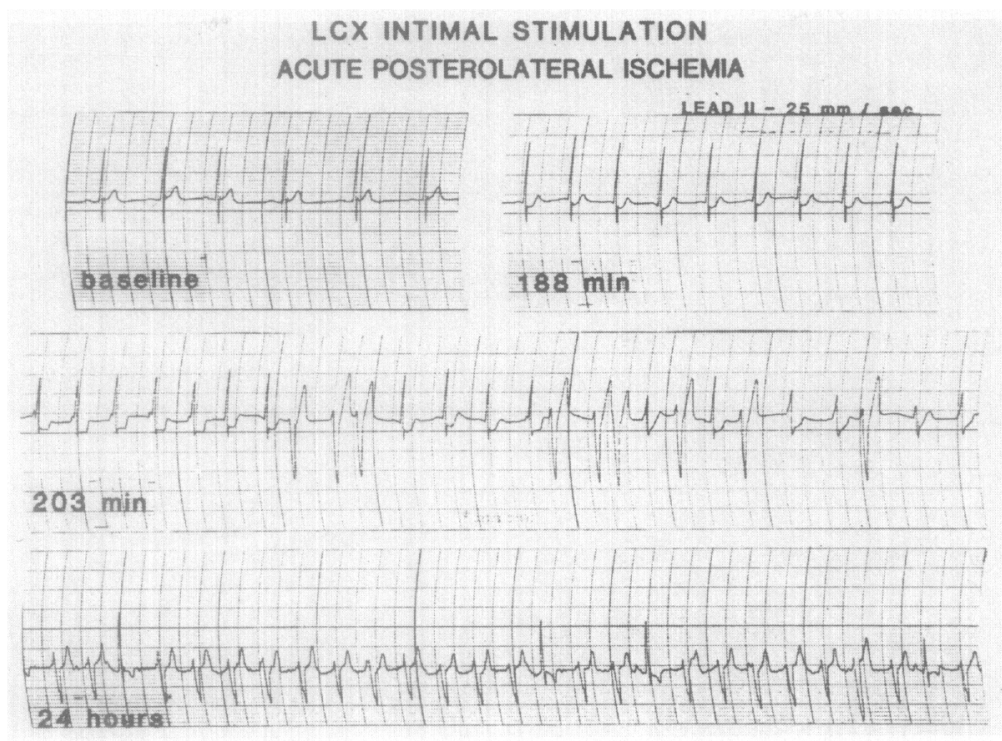

Fig. 5. In a noninducible animal with a small anterior infarction (4\% left ventricle), ST depression appeared after slightly more than 3 hours of current flow through the circumflex wire, followed by frequent premature ventricular contractions. After surviving 24 hours of ischemia, the animal had a large posterolateral infarct ( $28 \%$ left ventricle) and $80 \%$ of beats were ventricular in origin.

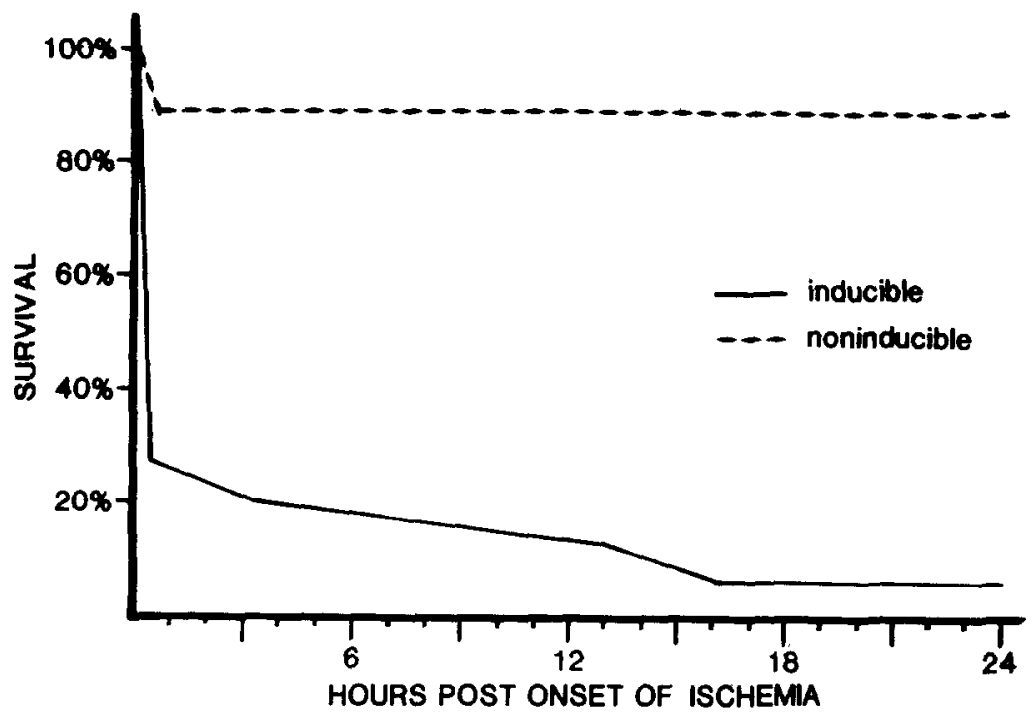

Fig. 6. Survival curves over the 24 hours following the onset of ECG evidence of ischemia.

cardia in this phase was observed rarely, however, and death occurred only in the inducible group. Representative tracings from a noninducible animal that survived 24 hours of ischemia are shown in Fig. 5. Survival rate at 24 hours for all animals was $7 \%$ in the inducible group and $87 \%$ in the noninducible group $(p<0.001)$. Fig. 6 depicts survival curves for the 24-hour period after the onset of ischemia.

Postmortem examination. All animals had intimal injury and thrombus formation at the site of the implanted circumflex coronary artery electrode. There was a trend toward greater thrombus mass in the noninducible group, although this was not statistically significant (Table II). It is likely that this trend reflects the longer survival time in the latter group.

The mass of infarcted tissue in the left anterior descending distribution, identified both by grossly visible infarcted tissue and the absence of histo- 


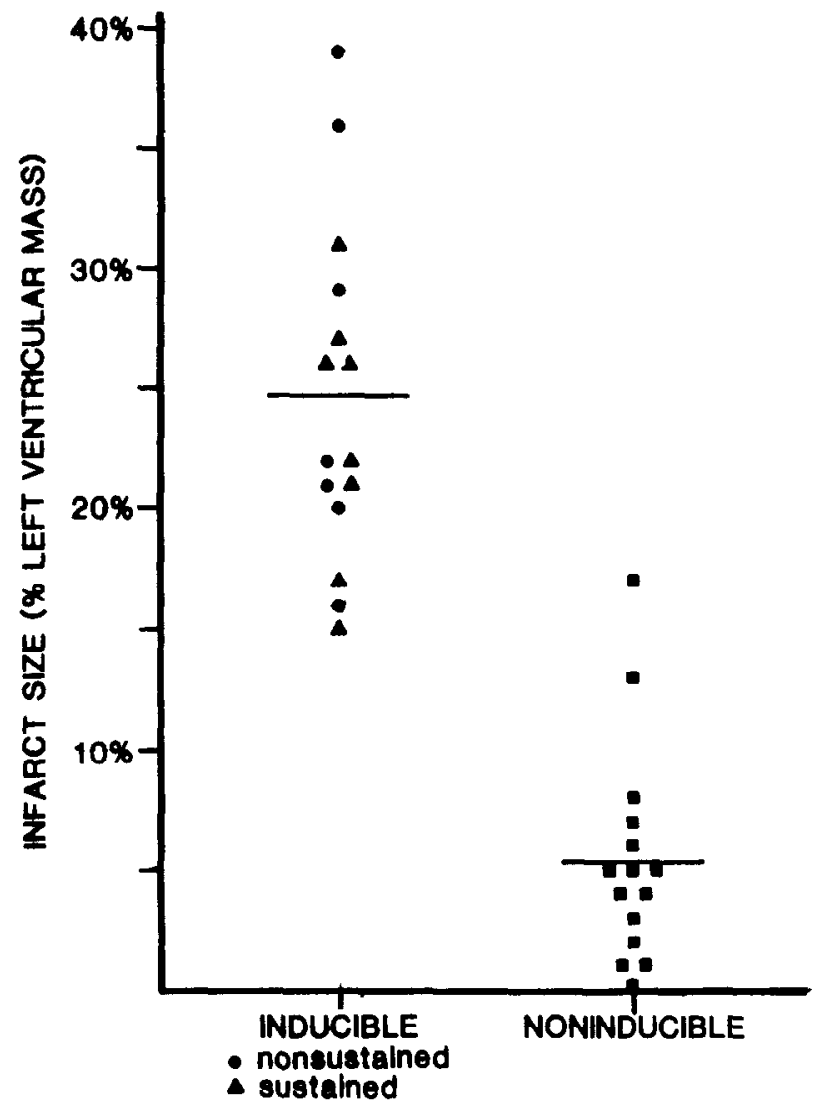

Fig. 7. The relationship of inducible ventricular tachycardia to anterior infarct size (percentage of left ventricular mass).

chemical staining, varied widely. In all animals the greatest area of infarction was subendocardial, although larger infarcts extended into the subepicardium and occasionally were transmural. However, infarct size clearly differentiated inducible from noninducible animals; inducible animals had much larger infarcts (Fig. 7). There was no difference in anterior infarct size between animals with inducible sustained ( $23.4 \pm 2.2 \%$ of the left ventricular mass) versus nonsustained $(26.1 \pm 3.3 \%$ of the left ventricular mass) ventricular tachycardia. If the data are analyzed by 24-hour survival, regardless of inducibility, nonsurvivors had significantly larger anterior infarcts $(23 \pm 2.6 \%$ vs $6.9 \pm 1.8 \%$, $p<0.01$ ). All other baseline characteristics did not differ between survivors and nonsurvivors.

All animals surviving more than 4 hours of ischemia had posterolateral infarcts identified by failure to stain with triphenyltetrazolium. The posterolateral infarct size was similar in surviving inducible as compared to noninducible animals (Table II). Posterolateral infarct size did not correlate with previous anterior infarct size $(\mathrm{r}=0.19, p=0.5)$.
Characteristics of animals excluded from the study. Six dogs developed ventricular fibrillation after induction of ventricular tachycardia during programmed stimulation. The anterior infarct size in these animals ( $25.0 \pm 2.9 \%$ of left ventricular mass) and all other baseline characteristics were not significantly different from animals with inducible ventricular tachycardia in which fibrillation did not occur. No animal that developed ventricular fibrillation had an anterior infarct of less than $15 \%$.

\section{DISCUSSION}

Previous investigators have established a relationship between pathologic and scintigraphic measures of infarct size in humans and subsequent overall mortality rates after acute myocardial infarction. ${ }^{19,20}$ The role of infarct size in influencing spontaneous and inducible arrhythmias in the convalescent phase is less well defined.

Inducible ventricular tachycardia. The ability to induce ventricular tachycardia was directly dependent upon the size of the recent infarct. Failure to find differences in infarct size between animals with sustained versus nonsustained ventricular tachycardia may be related to the protocol design, since no attempt was made to induce sustained arrhythmias once five or more nonstimulated ventricular complexes were obtained.

Experimental conditions may alter the relationship between infarct size and inducible arrhythmias. With the use of programmed stimulation from right ventricular sites in conscious dogs, others have reported findings similar to those of the present study ${ }^{21,22}$ However, investigators stimulating open chested anesthetized animals from multiple left ventricular sites could induce ventricular tachycardia even in animals with extremely small infarcts..$^{23,24}$ In two recent studies, the induction of ventricular tachycardia by programmed stimulation in the canine heart appeared to be a nonspecific response, not dependent upon previous injury, when stimulation was performed in open chest anesthetized animals. ${ }^{25,26}$ We were unable to induce ventricular tachycardia or ventricular fibrillation in any animal when the extent of myocardial damage was less than $10 \%$ of left ventricular mass. Previous studies in conscious animals support this finding. ${ }^{16,21,22}$ Alterations in autonomic tone, which play a significant role in arrhythmogenesis, ${ }^{27,28}$ may occur during surgery and anesthesia that facilitate tachyarrhythmia induction. It is doubtful that such arrhythmias are clinically relevant.

In humans, a similar relationship between infarct size and inducible arrhythmias may exist, although estimates of infarct size can only be inferred from 
indirect indices. Richards et al. ${ }^{12}$ studied 165 patients at an average of 10 days after infarction ( $55 \%$ of all patients presenting to that hospital, who survived the initial 5 days) and found that patients with inducible ventricular tachycardia had higher peak creatine phosphokinase plasma concentrations and lower mean ejection fractions than noninducible patients. Santarelli et al. ${ }^{14}$ found a higher incidence of severe wall motion abnormalities in postinfarct patients with inducible ventricular tachycardia. In the latter study and in that of Marchlinski et al., ${ }^{15}$ no differences in ejection fractions between inducible and noninducible patients were observed. Both studies involved smaller more select patient populations, studied with a less aggressive induction protocol. In addition, the majority of patients studied by Marchlinski et al. ${ }^{15}$ had inferior myocardial infarctions. In these patients, left ventricular ejection fraction would not reflect infarcted right ventricular myocardium.

In the present investigation, inducible ventricular tachycardia was highly predictive of spontaneous ventricular fibrillation during the early phase of a subsequent acute ischemic event. Infarct size was an equally powerful predictor of arrhythmic death. Animals with large anterior infarcts had both inducible ventricular tachycardia and died from ventricular fibrillation (not associated with hemodynamic collapse) within minutes of the onset of a secondary ischemic episode. Whether ventricular tachycardia in the early convalescent phase of myocardial infarction is simply a marker of infarct size or bears a closer mechanistic relationship to the occurrence of spontaneous ventricular fibrillation is unclear.

Sudden death during acute Ischemla. Previous investigations in anesthetized animals have established that the major risk of ventricular fibrillation occurs within the first 30 minutes of coronary occlusion. ${ }^{29,30}$ Thereafter, the incidence of ventricular fibrillation markedly diminishes despite continuing ischemia. A similar pattern was seen in the present study. Ventricular fibrillation generally was preceded by a slower, more organized ventricular rhythm, which subsequently accelerated after a variable period of time (usually less than a minute). This sequence of events has been noted in humans as well, when ventricular fibrillation was fortuitously recorded during Holter monitoring. ${ }^{31,32}$ The rate and morphology of the ventricular arrhythmia immediately preceding ventricular fibrillation was occasionally similar to that previously induced by programmed stimulation, suggesting a common mechanism. More often, there were no clear similarities between the two.

Studies during acute ischemia in anesthetized animals without previous infarction suggest three other variables, which influence the risk of early ventricular fibrillation-the site of occlusion relative to the origin of the artery, ${ }^{33}$ heart rate, ${ }^{34-36}$ and the presence of preexisting collaterals. ${ }^{37}$ All of these factors indirectly influence the size of the acutely ischemic area. The work of Endo et al., ${ }^{38}$ which used injected microspheres 1 minute after the onset of coronary occlusion, suggests that differences in the size of the acute ischemic zone may partially, but not completely, account for differences in the risk of ventricular fibrillation. These investigators found only a small, although statistically significant, difference in hypoperfused myocardial mass between those animals which fibrillated and those that did not $(31.6 \%$ vs $26.3 \%$ of left ventricular mass). In addition, faster heart rates are associated with progressively impaired conduction and prolonged refractoriness in both acutely ischemic $c^{34,35}$ and chronically injured muscle..$^{39,40}$ The cycle lengthdependent changes may also predispose to lethal arrhythmias.

In this investigation, the site of occlusion was constant and both blood pressure and maximum heart rate during ischemia were similar for those animals that fibrillated and those that survived. We could not quantitate the mass of acutely ischemic myocardium in animals dying within minutes after the onset of ischemia. In survivors, the lack of correlation between anterior infarct size and ultimate posterolateral infarct size suggests that there were no systematic differences between inducible and noninducible animals with regard to the size of the posterolateral hypoperfused zone.

Type of infarction. This study specifically examined the vulnerability to late arrhythmias in infarctions produced by temporary occlusion. These infarcts differ morphologically and electrophysiologically from those produced by permanent occlusion. The ultimate size of infarction produced by reperfusion may be the same or smaller than that produced by permanent occlusion at the same level. ${ }^{21}$ However, reperfusion infarctions have a wider rim of endocardial and particularly epicardial myocardium that is spared. ${ }^{21,41,42}$ The infarct itself is mottled and encompasses islands of normal tissue interspersed with necrotic or ischemic cells. Permanent occlusion infarction in dogs produces a thinner rim of surviving epicardial myocardium, and within the infarction there is homogeneous necrosis. On the endocardium, only a few layers of Purkinje cells survive, and virtually no viable myocardial cells can be identified. . $2,43^{43}$

Programmed ventricular stimulation can induce ventricular tachycardia in both types of models 
several days to weeks after infarction, ${ }^{16,21,23,24,39}$ but such arrhythmias may be more easily induced after reperfusion infarction. ${ }^{21}$ In both models, surviving fibers adjacent to the infarct possess abnormal action potential characteristics and responses to stimulation when studied in vitro days to weeks after the infarction. However, the distribution of these fibers is predominantly limited to the epicardium in permanent occlusion models, ${ }^{40,44,45}$ while they occur both epicardially and endocardially, and probably intramurally as well, in reperfusion infarcts. ${ }^{41.42,45}$ Richards et al..$^{46}$ have demonstrated a wide variability of local extracellular refractory periods and excitability throughout all layers of the reperfusion infarct compared to the noninfarcted region of the ventricle. These differences both in extent and distribution of electrophysiologically abnormal tissue may play an important role in subsequent genesis of arrhythmias.

Infarct morphology in humans is heterogeneous. ${ }^{47-49}$ Those infarcts involving predominantly the subendocardium are distinctive in that there is a greater frequency of patchy rather than uniform necrosis, a higher incidence of contraction band necrosis, and a markedly lower incidence of total occlusion of the infarct-related vessel. These findings suggest transient interruption of flow rather than prolonged occlusion as an etiologic factor, ${ }^{47}$ similar to canine reperfusion infarction. The electrophysiologic properties of these infarcts in humans have not been studied, although sudden death occurs frequently..$^{47,49} \mathrm{In}$ addition, Malacoft et al. ${ }^{50}$ demonstrated an increased incidence of inducible ventricular tachycardia in the convalescent phase of infarction in those patients who were successfully reperfused within 3 hours of symptom onset relative to patients in whom reperfusion was unsuccessful. The findings of this study may be most applicable to those patients who undergo early spontaneous or therapeutic reperfusion.

Timing of observations. The observations in the present investigation were made in the early convalescent phase of infarction. While of obvious clinical importance, the question of whether or not the demonstrated relationships between infarct size, inducible ventricular tachycardia, and spontaneous ventricular fibrillation remains valid over longer periods of time remains to be determined. Earlier studies from this laboratory demonstrated that ventricular tachycardia could be initiated reproducibly up to 2 weeks after reperfusion infarction..$^{51}$ The high risk of ventricular fibrillation after an acute ischemic event was present even 1 month after infarction..$^{52}$ Investigations over a more extended period of time have not been performed.
There may be significant changes in the electrophysiologic properties of infarcted tissue after healing is completed. In humans, this process may be considerably prolonged relative to canine models and may be dependent, in part, on infarct size. ${ }^{42}$ In canine models, detailed studies of surviving ventricular muscle fibers (as opposed to Purkinje fibers) more than 3 weeks after infarction are lacking. In cats, Myerburg et al. ${ }^{53}$ have demonstrated persistently abnormal action potentials and local refractory periods in subendocardial tissue up to 6 months after permanent occlusion of distal branches of the anterior descending artery. It should be added that the propensity for late reentrant arrhythmias may not depend solely upon demonstrable abnormalities of individual fibers (abnormal membrane properties) but may also be affected by resistance changes between cells because of fibrosis and patchy necrosis. ${ }^{54}$ Clearly, more information is needed, both in humans and experimental animals, before the long. term implications of this study can be assessed.

Ischemia as a triggering event. In the present study, we used acute ischemia to precipitate ventricular fibrillation. Other triggering mechanisms, particularly abrupt changes in wall stress or tension ${ }^{55}$ and changes in autonomic tone, ${ }^{27}$ may play important roles in the genesis of postinfarction sudden death. Both are altered during the early phases of ischemia as well.

Potential influence of infarct size on late arrhythmias. Past investigators have stressed the importance of nonuniform recovery of excitability in the genesis of ventricular fibrillation. ${ }^{56,57}$ In the convalescent canine infarction, heterogeneous recovery properties-as reflected by delayed activation and temporal dispersion of refractoriness-have been documented. Janse et al. ${ }^{58,59}$ have mapped spontaneous reentrant rhythms in the in situ regionally ischemic pig heart. In general, the reentrant wave front circulated around an area of conduction block, which was only 1 or $2 \mathrm{~cm}$ in diameter. Tachycardias that terminated spontaneously maintained these relatively large dimensions. When ventricular fibrillation ensued, the activation front encountered numerous areas of block and became fractionated into multiple circuits, frequently incomplete, with much smaller diameters of revolution (a few millimeters).

The presence of previously infarcted myocardium may facilitate both the onset of reentrant tachycardia and further fractionation of a circulating wave front. Premature beats arising from within or at the border of the ischemic zone would encounter a variable region of greatly heterogeneous recovery properties (perhaps more than within the acutely 
ischemic area itself). The greater the mass of this region, the more likely that sufficiently slowed conduction and an area of functional block will develop while the remaining myocardium recovers enough to sustain reexcitation. Successive wave fronts from a reentrant tachycardia arising within acutely ischemic myocardium may undergo further fractionation as it encounters previously infarcted tissue (particularly as cycle lengths shorten). The greater the mass of previously injured myocardium through which these premature activation fronts must pass, the more likely that a critical degree of fractionation, and thus fibrillation, may occur.

Conclusions. In a conscious canine model, we have demonstrated that the extent of previous myocardial injury plays a critical role in influencing vulnerability to both inducible ventricular tachycardia and spontaneous ischemia-related ventricular fibrillation several days later. These findings support the importance of infarct size limitation in reducing the mortality rate after myocardial infarction. However, if a significant mass of irreversibly injured myocardium persists after early therapeutic reperfusion, such interventions may not alter the subsequent risk of arrhythmic death.

\section{REFERENCES}

1. Reichenbach DD, Moss NS, Meyer E: Pathology of the heart in sudden cardiac death. Am J Cardiol 39:865, 1977.

2. Roy D, Waxman HL, Kienzle MG, Buxton AE, Marchlinski FE, Josephson ME: Clinical characteristics and long-term follow-up in 119 survivors of cardiac arrest. Am J Cardiol 52:969, 1983.

3. Ruskin JN, Garan H: Electrophysiologic observations in survivors of out-of-hospital cardiac arrest related to ischemic heart disease. Cardiovasc Clin 1:287, 1983.

4. Mukharji J, Rude RE, Poole WK, Gustafson N, Thomas LJ, Strauss HW, Jaffe AS, Muller JE, Roberts R, Raabe DS, Croft CH, Passamani E, Braunwald E, Willerson JT: Ventricular arrhythmia and left ventricular dysfunction six months after myocardial infarction as risk factors for sudden death. Circulation 68:106, 1983.

5. Gilpin EA, Koziol JA, Madsen EB, Henning H, Ross J: Periods of differing mortality distribution during the first year after myocardial infarction. Am $J$ Cardiol 52:240, 1983.

6. Schulze RA, Strauss HW, Pitt B: Sudden death in the year following myocardial infarction. Am J Med 62:192, 1977.

7. Moss AJ, Davis HT, DeCamilla J, Bayer LW: Ventricular ectopic beats and their relation to sudden and nonsudden cardiac death after myocardial infarction. Circulation 60:998, 1979.

8. Olson HG, Lyons KP, Troop P, Butman S, Piters KM: The high-risk acute myocardial infarction patient at 1-year follow-up. Am Heart J 107:358, 1984.

9. Schuster EH, Bulkley BH: Early post-infarction angina. N Engl J Med 305:1101, 1981.

10. Theroux P, Waters DD, Halphen C, Debaisieux J, Mizgala HF: Prognostic value of exercise testing soon after myocardial infarction. N Engl J Med 301:341, 1979.

11. Hamer A, Vohra J, Hunt D, Sloman G: Prediction of sudden death by electrophysiologic studies in high risk patients surviving acute myocardial infarction. Am J Cardiol 50:223, 1982.
12. Richards DA, Cody DV, Denniss AR, Russell PA, Young AA, Uther JB: Ventricular electrical instability: A predictor of death after myocardial infarction. Am $J$ Cardiol 51:75, 1983.

13. Marchlinski FE, Buxton AE, Waxman HL, Josephson ME: Identifying patients at risk of sudden death after myocardial infarction. Am J Cardiol 52:1190, 1983.

14. Santarelli P, Bellocci F, Loperfido F, Mazzari M, Mongiardo $R$, Denes P, Gemelli PA: Ventricular electrical instability in acute myocardial infarction. Circulation 68:108, 1983.

15. Marchlinski FE, Waxman HL, Buxton AE, Josephson ME: Sustained ventricular tachyarrhythmias during the early post infarction period. J Am Coll Cardiol 2:240, 1983.

16. Patterson E, Holland K, Eller BT, Lucchesi BR: Ventricular fibrillation resulting from ischemia at a site remote from previous infarction. Am J Cardiol 50:1414, 1982.

17. Sheehan FH, Epstein SE: Determinants of arrhythmic death due to coronary spasm: Pre-existing stenosis reduces risk of VF following release of coronary occlusion. Circulation 56:259, 1982.

18. Fishbein MC, Meerbaum S, Rit J, Lendo U, Kanmatsuse K, Mercier JC, Corday E, Ganz W: Acute phase early myocardial infarct size quantitation: Validation of the triphenyltetrazolium chloride tissue enzyme slaining technique. Am Heart J 101:593, 1981.

19. Cabin HS, Roberts WC: Relation of healed transmural infarct size to length of survival after acute myocardial infarction, age at death, and amount and extent of coronary arterial narrowing by atherosclerotic plaques. AM HEART J 104:216, 1982.

20. Silverman KJ, Becker LC, Bulkley BH, Burow RD, Mellits ED, Kallman CH, Weisfelt ML: Value of early thallium-201 scintigraphy for predicting mortality in patients with acute myocardial infarction. Circulation 61:996, 1980.

21. Kuragueuzian HS, Fenoglio JJ, Weiss MB, Wit AL: Protracted ventricular tachycardia induced by premature stimulation of the canine heart after coronary artery occlusion and reperfusion. Circ Res 44:833, 1979.

22. Jones-Collins BA, Patterson RE: Quantitative measurement of electrical instability as a function of myocardial infarct size in the dog. Am J Cardiol 48:858, 1981.

23. Michelson EL, Spear JF, Moore EN: Electrophysiologic and anatomic correlates of sustained ventricular tachyarrhythmias in a model of chronic myocardial infarction. Am $J$ Cardiol 45:583, 1980.

24. Garan H, Fallon JT, Ruskin JN: Sustained ventricular tachycardia in recent canine myocardial infarction. Circulation 62:980, 1980.

25. Wetstein L, Michelson EL, Simson MB, Moore EN, Harken AH: Initiation of ventricular tachyarrhythmia with programmed stimulation: Sensitivity and specificity in an experimental canine model. Surgery 92:206, 1982.

26. Echt DS, Griffin JC, Ford AJ, Knutti JW, Feldman RC, Mason JW: Nature of inducible ventricular tachyarrhythmias in a canine chronic myocardial infarction model. Am J Cardiol 52:1127, 1983.

27. Corr PB, Gillis RA: Autonomic neural influences on the dysrhythmias resulting from myocardial infarction. Circ Res 43:1, 1978.

28. Rardon DP, Bailey JC: Parasympathetic effects on electrophysiologic properties of cardiac ventricular tissue. J Am Coll Cardiol 2:1200, 1983.

29. Harris AS, Rojas AG: The initiation of ventricular fibrillation due to coronary occlusion. Exp Surg 1:105, 1984.

30. Kaplinsky E, Ogawa S, Balke CW, Dreifus LS: Two periods of early ventricular arrhythmias in the canine acute myocardial infarction model. Circulation 60:397, 1979.

31. Nikolic R, Bishop RL, Singh JB: Sudden death recorded during Holter monitoring. Circulation 66:218, 1982.

32. Panidis IP, Morganroth J: Sudden death in hospitalized patients: Rhythm disturbances detected by ambulatory electrocardiographic monitoring. J Am Coll Cardiol 2:798, 1983. 
33. Allen JB, Laadt JR: The effect of the level of the ligature on the mortality following ligation of the circumflex coronary artery in the dog. AM HEART J 39:273, 1950.

34. Scherlag BJ, El-Sherif N, Hope R, Lazzara R: Characterization and localization of ventricular arrhythmias resulting from myocardial ischemia and infarction. Circ Res 35:372, 1974.

35. Hope RR, Williams DO, El-Sherif N, Lazzara R, Scherlag BJ: The efficacy of antiarrhythmic agents during acute myocardial ischemia and the role of heart rate. Circulation 50:507, 1974.

36. Fujimoto 'T, Peter 'T, Hamamoto H, Mandel WJ: Electrophysiologic observations during the spontancous initiation of ischemia-induced ventricular fibrillation. AM HEART J 105:189, 1983.

37. Meesmann W: Early arrhythmias and primary ventricular fibrillation after acute myocardial ischemia in relation to pre-existing coronary collaterals. In Parratt JR, editor: Early arrhythmias resulting from myocardial ischemia. New York. 1982, Oxford University Press, p 93.

38. Endo T, Ribeiro LG, Cheung W, Faria DB, Petranto M, Maroko PR: Relationship between the extent of the hypoperfused zone of the myocardium and the occurrence of ventricular fibrillation. AM HEART $J$ 105:915, 1983.

39. Hope RR, Scherlag BJ, El-Sherif N, Lazzara R: Ventricular arrhythmias in healing myocardial infarction. J Thorac Cardiovasc Surg 75:458, 1978 .

40. El-Sherif N, Scherlag BJ, Lazzara R, Hope RR: Re-entrant ventricular arrhythmias in the myocardial infarction period. 1. Conduction characteristics in the infarction zone. Circulation 55:686, 1977.

41. Karagueuzian HS, Fenoglio JJ, Weiss MB, Wit AL: Coronary occlusion and reperfusion: Effects on subendocardial cardiac fibers. Am J Physiol (Heart) 238:H581, 1980.

42. Ursell PC, Fenoglio JJ: Structural basis of ventricular tachy cardia. In Josephson ME, editor: Ventricular tachycardia. Mt. Kisco, 1982, Futura, p 151.

43. Friedman PL, Stewart JR, Fenoglio JJ, Wil AL: Survival of subendocardial Purkinje fibers after extensive myocardial infarction in dogs. Circ Res 33:597, 1973.

44. Gardner P, Ursell PC, Fenoglio JJ, Allessie MA, Bonke FI, Wit AL: Structure of the epicardial border zone in canine infarcts as a cause of re-entrant excitation. Circulation 64:320, 1981.

45. Wit AL: Electrophysiologic mechanisms of ventricular tachycardia caused by myocardial ischemia and infarction in experimental animals. In Josephson ME, editor: Ventricular tachycardia. Mt. Kisco, 1982, Futura, p 33.
46. Richards DA, Blake GJ, Spear JF, Moore EN: Electrophysiologic substrate for ventricular tachycardia: Correlation of properties in vivo and in vitro. Circulation 69:369, 1984.

47. Freifeld AG, Scht ster EH, Bulkley BH: Nontrasmural versus transmural myocardial infarction: A morphologic study. Am J Med 75:423, 1983 .

48. Baroldi G, Radice F, Schmid G, Leone A: Morphology of acute myocardial infarction in relation to coronary thrombosis. Am Heart J 87:65, 1974.

49. Erhardt LR: Clinical and pathological observations in different types of acute myocardial infarction. Acta Med Scand (Suppl 6) 560:7, 1974.

50. Malacoff R, McGovern B, Ruskin J, Gold H, Leinbach R, Kelly E, Garan H: Ventricular electrical instability in patients treated with streptokinase during acute myocardial infarction. Am J Coll Cardiol 1:604, 1983 .

51. Patterson E, Gibson JK, Lucchesi BR: Postmyocardial infarction re-entrant arrhythmias in conscious dogs: Suppression by bretylium tosylate. J Pharmacol Exp Ther 216:453, 1981.

52. Patterson E, Eller BT, Abrams GD, Vasiliades J, Lucchesi BR: Ventricular fibrillation in a conscious canine preparation of sudden coronary death-prevention by short and long term amiodarone administration. Circulation 68:857, 1983.

53. Myerburg RJ, Epstein K, Gaide MS, Wong SS, Castellanos A, Gelband H, Bassett AL: Electrophysiologic consequences of experimental acute ischemia superimposed upon healed myocardial infarction in cats. Am J Cardiol 49:323, 1982.

54. Spach MS, Kootsey JM: The nature of electrical propagation in cardiac muscle. Am J Physiol 244:H3, 1983.

55. Lab MJ: Contraction-excitation feedback in myocardium: Physiologic hasis and clinical relevance. Circ Res 50:757, 1982.

56. Han J, Moe GK: Nonuniform recovery of excitability in ventricular muscle. Circ Res 14:44, 1964.

57. Merx W, Yoon MS, Han J: The role of local disparity in conduction and recovery time on ventricular vulnerability to fibrillation. AM HEART J 94:603, 1977.

58. Janse MJ, Kleber AG: Electrophysiological changes and ventricular arrhythmias in the early phase of regional myocardial ischemia. Circ Res 49:1069, 1981.

59. Janse MJ, Van Capelle FJ, Morsink H, Kleber AG, WilmsSchopman F, Cardinal R. D'Alnoncourt CN, Durrer D: Flow of injury current and patterns of excitation during early ventricular arrhythmias in acute regional myocardial ischemia in isolated porcine and canine hearts. Circ Res 47:151, 1980. 